\title{
CHARGE-NEUTRALIZATION ELECTRODES FOR HIGH-TENSION SEPARATORS
}

\author{
L. Dascalescu, R. Morar, Al. Iuga, V. Neamtu, I. Suarasan \\ Technical University of Cluj-Napoca, 15 C. Daicoviciu Street, \\ 3400 Cluj-Napoca, Romania
}

(Received May 25, 1992)

\begin{abstract}
Safe and efficient operation of industrial high-tension separators requires a strict control of residual electric charge of the particles emerging the process. Principles of charge neutralization in a rolltype electroseparator are briefly discussed, in order to establish the general features of the corona electrodes that might accomplish this task. A new electrode design is suggested. Its spacific features: small cross-section (neglectable perturbation of the material stream), low corona on-set voltage, good stability of corona discharge during long-time operation, short maintenance time, have been tested on a laboratory model, prior to industrial application. Several design considerations have been formulated, based on a critical evaluation of the experimental results.
\end{abstract}

\section{INTRODUCTION}

Electroseparation technologies [1], [2] are based on the electric forces acting on charged or polarized bodies. This is why most researches in this domain focused on charging or polarizing mechanisms, the efficiency of which determines the quality of the products [3], [4]. Little has been written [5] on the methods to neutralize the residual charge of the particles at the exit from an electroseparation process.

High-conductivity particles transfer fast their charge to the earthed metallic parts of a high-tension separator. The charged low-conductivity objects are much more difficult to handle. 
Such particles are "pinned" to the earthed rotating roll electrode by the electric image force $F_{i}$ (Fig. 1). They can preserve their charge long after the moment when they are mechanically brushed-off the surface of the roll carrier. The accumulation of charged granules in the collecting hoppers of the electroseparators should be avoided, because: (i) the flow of the material is hindered by the particles being pinned to the metallic duct, due to the electric image force; (ii) any charged particle approaching a metallic wall may initiate a local breakdown of the gas. This second argument becomes extremely important when processing easycombustible materials (foodstuffs, textile fibers, cellulose).

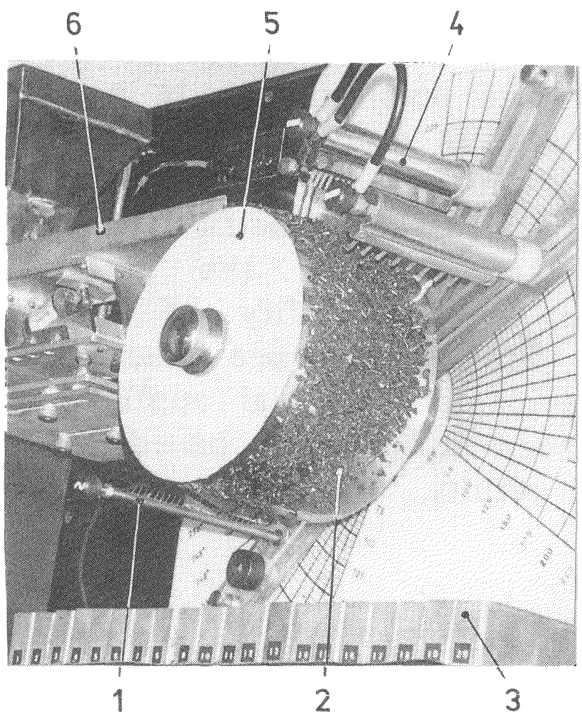

a

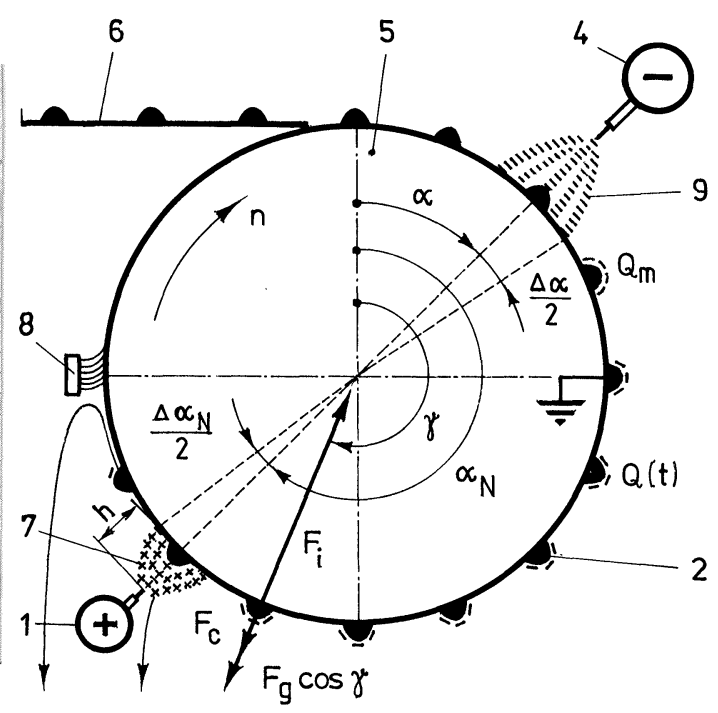

$b$

Fig. 1. "Pinning" of charged low-conductivity particles on the surface of the earthed rotating roll (a); Variables which characterize the process of corona charging and charge neutralization in a rolltype separator (b); 1 - corona electrode for charge neutralization (wiper electrode); 2 - non-conductive particle; 3 - collecting boxes; 4 - active corona electrode; 5 - earthed rotating roll electrode; 6 vibratory feeder; 7 - zone of positive space charge generated by the wiper electrode; 8 - brush; 9 - zone of negative space charge generated by the active electrode. 
The present paper discusses the general features of the corona electrodes that could be employed for charge neutralization in rolltype high-tension separators. It introduces an original electrode design, the characteristics of which are presented in detail.

\section{CHARGE NEUTRALIZATION IN A ROLL-TYPE HIGH-TENSION SEPARATOR}

Charging or discharging of a semi-ellipsoidale particle of axes a and b (Fig. 2), in contact with an elctrode affected by the uniform electric field $\mathrm{E}$ and by the density of the corona current $\mathrm{J}$, can be expressed as:

$$
\mathrm{Q}(\mathrm{t})=\pi \cdot \mathrm{b}^{2} \cdot \mathrm{q}(\mathrm{t})
$$

where $q(t)$ satisfies the equation:

$\mathrm{dq}(\mathrm{t}) / \mathrm{dt}=-\mathrm{q}(\mathrm{t}) / \tau+[\sigma-(\mathrm{J} / \mathrm{E}) \cdot(1+\chi)] \cdot \mathrm{E} /(1+\mathrm{N} \cdot \chi)$,

$\sigma$ - electrical conductivity of the particle,

$\chi$ - electrical susceptibility of the particle,

$\mathrm{N}$ - depolarization factor (function of $\mathrm{a} / \mathrm{b}[6]$ ),

$\tau$ - time constant, calculated as follows:

$\tau=\left\{\mathrm{J} .(1-\mathrm{N}) /\left[\varepsilon_{0} . \mathrm{E} \cdot(1+\mathrm{N} \cdot \chi)\right]+\mathrm{N} \cdot \sigma /\left[\varepsilon_{0} \cdot(1+\mathrm{N} \cdot \chi)\right]\right\}^{-1}$.

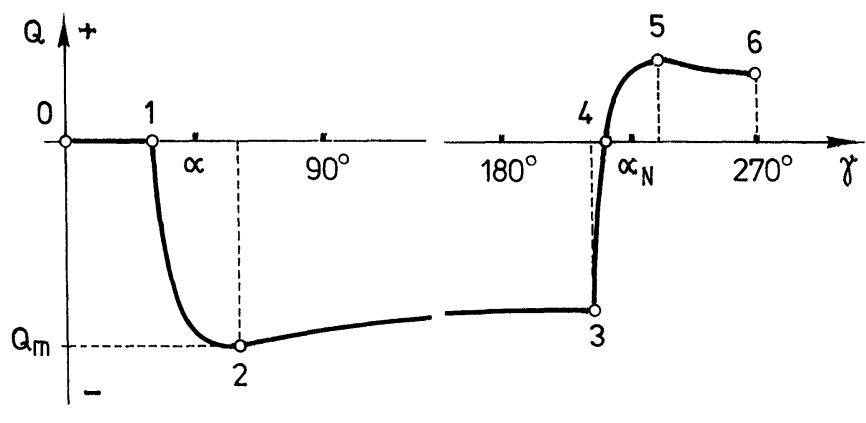

a

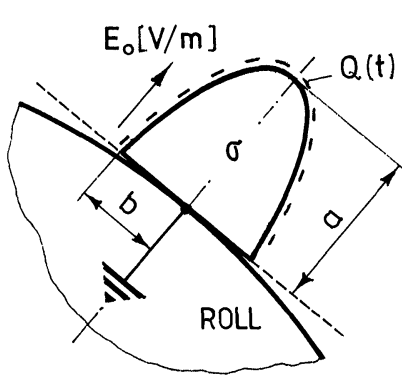

b

Fig. 2. Qualitative representation of charge variation during the process of negative d. c. corona charging $(1-2)$, charge decaying (2 - 3), charge neutralization ( 3 - 4), positive d. c. corona charging (4 5) (a), in the case of a low-conductivity particle, assimilated to a hemi-ellipsoid of revolution (b). 
In the case of a low-conductivity particle, the time constant of the process is:

$$
\tau_{\text {charge }}=\varepsilon_{0} . \mathrm{E} .(1+\mathrm{N} . \chi) /[\mathrm{J} .(1-\mathrm{N})] .
$$

Considering $\mathrm{J}=10^{-2} \mathrm{~A} / \mathrm{m}, \mathrm{E}=5 \mathrm{kV} / \mathrm{cm}, \mathrm{N}=0.2, \chi=2, \varepsilon_{0}=10^{-11}$ $\mathrm{F} / \mathrm{m}$, one can evaluate:

$$
\tau_{\text {charge }}=8.7510^{-4} \mathrm{~s}<1 \mathrm{~ms} .
$$

The discharging process $-\mathrm{J}=0$ in equation (3) - is very slow:

$$
\tau_{\text {discharge }}=\varepsilon_{0} .(1+\mathrm{N} . \chi) /(\mathrm{N} . \sigma)=700 \mathrm{~s},
$$

as compared to the duration $\tau_{t}$ of particle transfer from the charging zone $(\alpha=\pi / 4)$ to the charge-neutralization zone $\left(\alpha_{N}=\right.$ $5 \pi / 4)$, if the velocity of the rotating roll electrode is $\mathrm{n}=2 \mathrm{rot} / \mathrm{s}$ :

$$
\tau_{\mathrm{t}}=\left(\alpha_{\mathrm{N}}-\alpha\right) /(2 . \pi . \mathrm{n})=[(5 \pi / 4)-(\pi / 4)] /(2 \pi .2)=0.25 \mathrm{~s} .
$$

This means that the charge of the particle when entering the neutralization zone is almost equal to the saturation value [6]:

$\mathrm{Qm}=-(1+\chi) \cdot \pi \cdot \mathrm{b}^{2} \cdot \varepsilon_{0} \cdot \mathrm{E} /(1-\mathrm{N})$.

A primary objective of charge neutralization is to reduce the electric image force:

$$
F_{i}=Q^{2} /\left(4 . \pi . \varepsilon_{0} \cdot d^{2}\right),
$$

so that the particles detach from the rotating roll electrode ( $d$ represents the fictituous distance between $Q$ and the image charge, -Q). This is why the corona electrodes employed for charge neutralization are usually called "wiper electrodes".

Laboratory experiments demonstarted that d. c. or a. c. highvoltage supplies can be equally employed to energize these electrodes (Fig.3). In the first case, the polarity of the d. c. corona discharge generated by the wiper electrode is opposite to that of the active electrodes. The "ion bombardment" determines a fast neutralization of the cahrge and the detachement of the particles from the rotating roll electrode of radius $R$, as soon as:

$$
\mathrm{Q}<\left[4 . \pi \cdot \varepsilon_{0} \cdot \mathrm{d}^{2} \cdot\left(\mathrm{m} \cdot \mathrm{g} \cdot \cos \gamma+4 . \pi^{2} \cdot \mathrm{m} \cdot \mathrm{n}^{2} \cdot \mathrm{R}\right)\right]^{1 / 2},
$$

where $m$ is the mass of the particle. The radial extension of the neutralization zone should be:

$$
\Delta \alpha_{N}<5 . \tau_{\text {charge }} \cdot(2 . \pi . n)=510^{-3} \cdot 2 . \pi .2=\pi / 50,
$$

a condition that is very easy to accomplish.

A thorough study of the most-probable particle trajectories is necessary [7], in order to establish the position of the wiper electrode. The transit of detached, uncharged particles through the corona field generated by the wiper electrode should be avoided as 
much as posible. Otherwise, it is vary likely that the particles would charge again, before being collected in the product hoppers.

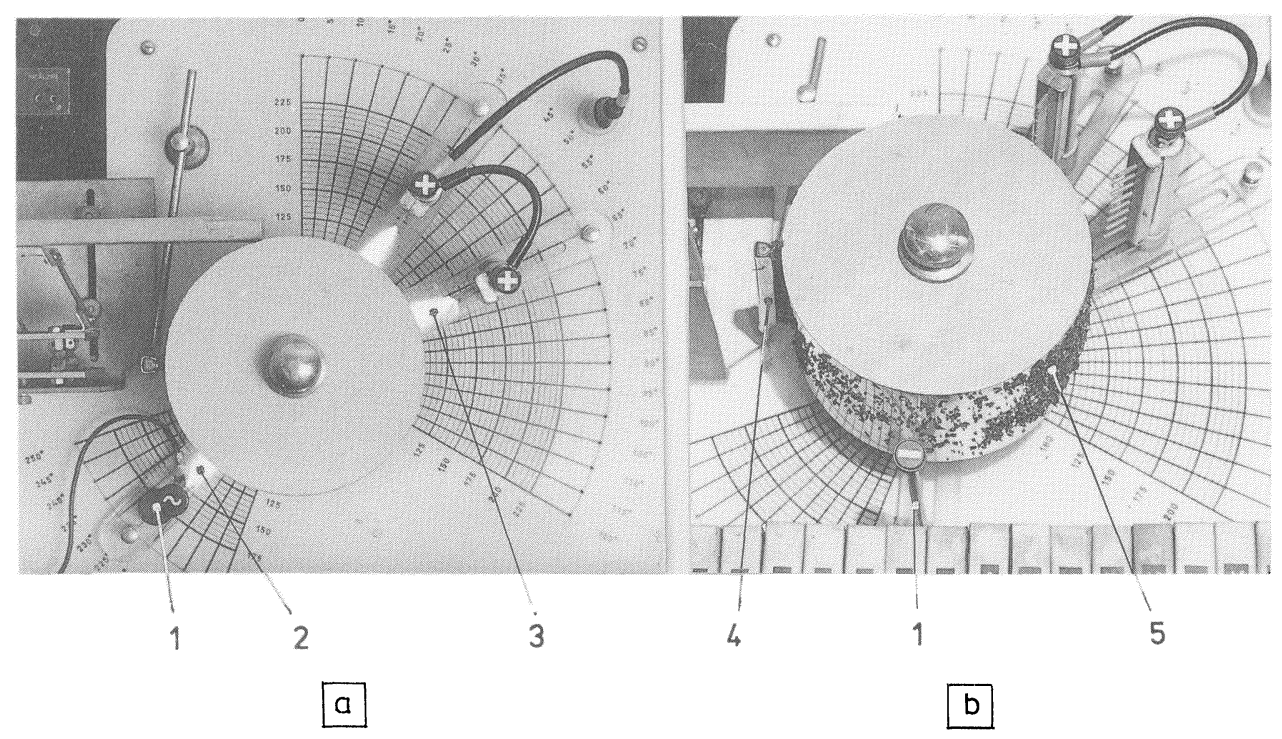

Fig. 3. Visualization of corona discharges produced by two positivepolarity active electrodes and by one electrode for charge neutralization, connected to a high-voltage generator $(0 \ldots 25) \mathrm{kV}$, a.c., $50 \mathrm{~Hz}$ (a); "wipping" effect produced by a negative-polarity electrode for charge neutralization (b); 1 - charge-neutralization electrode; 2 - alternating charge-neutralization corona field; 3 positive d. c. corona field; 4 - brush; 5 - layer of non-conducting granules, before entering the charge-neutralization field zone.

This situation is less critical in the case that an a. c. supply is employed to energize the wiper electrode, although the analysis of the charge neutralization phenomena is more complicated. If the "active" electrodes are of negative polarity, and if the charged particles approach the wiper electrode during the positive alternance of the corona discharge, the neutralization of their charge is produced as described above, except for the fact that:

$\mathrm{E}=\mathrm{E}(\mathrm{t})=\mathrm{E}_{\mathrm{m}} \cdot \sin (2 \cdot \pi . \mathrm{f} . \mathrm{t})$,

$f$ being the frequency of the high-voltage supply.

If the particle enter the a. c. corona discharge zone during the negative alternance, they increase - at first - their residual charge, which -anyhow - can not exeed the maximum value given by (8). 
As the voltage changes its sign, the positive alternance of the corona discharge produces a diminution of the charge of the particle. Thus, in order to achieve charge neutralization, the frequency of the high-voltage supply of the wiper electrode should be:

$$
\mathrm{f}<1 /\left(2 \cdot \tau_{\text {charge }}\right)=(1 / 2) \cdot 10^{3}=500 \mathrm{~Hz} \text {. }
$$

A $50 / 60 \mathrm{~Hz}$ high-voltage transformer can be successfully employed with industrial wiper electrodes. Let $\Delta \alpha_{N}=\pi / 6$ be the angular extension of the space charge generated by the wiper electrode, and $t_{N}$ the duration of particle transit through the charge-neutralization field zone. Imposing the condition: $t_{N}=1 / f$, and $\mathrm{f}=50 / 60 \mathrm{~Hz}$, the charge neutralization can be achieved if the angular velocity of the roll electrode were:

$$
2 . \pi . \mathrm{N}<\Delta \alpha_{\mathrm{N}} / \mathrm{t}_{\mathrm{N}}=\Delta \alpha_{\mathrm{N}} . \mathrm{f}=\pi . \mathrm{f} / 6 .
$$

Consequently:

$$
\begin{aligned}
& \mathrm{n}_{(50 \mathrm{~Hz})}<\mathrm{f} / 12=5 \mathrm{rot} / \mathrm{s}=300 \mathrm{rot} / \mathrm{min}, \\
& \mathrm{n}_{(50 \mathrm{~Hz})}<\mathrm{f} / 12=25 / 6 \mathrm{rot} / \mathrm{s}=250 \mathrm{rot} / \mathrm{min} .
\end{aligned}
$$

One can conclude that a high-voltage generator of $\mathrm{f}(0 \ldots 100) \mathrm{Hz}$ could ensure an effective a. c. charge-neutralization over the entire speed range $(0 \ldots 600) \mathrm{rot} / \mathrm{min}$ of usual high-tension separators.

\section{A NEW TYPE OF WIPER ELECTRODE}

Besides the electrical requirements discussed in the above section, a good charge-neutralization electrode has to satisfy several mechanical constraints, due to the fact that its position in an electroseparator inevitably affects the flow of the material. The usual design of dual corona electrodes, that is adequate to generate the main electric field zone of a high-tension separator [8], [9], can not be employed with wiper electrodes, because of its relativelylarge electrostatic element, which "collects" the electroinsulating particles emerging from the rotating roll electrode, hindering them to enter into the regular material stream.

If a dual type design is considered for a wiper electrode, then the cross-section of the electrostatic element should be reduced to a minimum, so that to prevent the build-up of a undesirable layer of material on its surface exposed to the flow of low-conductivity 
garnules. A wire-type corona element is not recommended, because it could be easily damaged by the stream of granular materials. The use of needles as corona-emitting elements is a better solution, but thay must be periodically changed because of wear, a requirement which is not very agreeable, from the operator's point of view.

This is why a new electrode design is suggested in Fig. 4. The tips of $15 \mathrm{~mm}$ - long segments of metallic wire 1 represent the corona-emitting elements. Each of these wire segments is introduced in a tubular metallic support 2. In spite of the fact that the corona-emitting elements are made of metals which are characterized by an elevated melting temperature (such as wolfram or inoxidable steel), the length of the wire segments reduces during operation, due to the material transportation which accompanies electric discharges from their tips. Therefore, periodical adjustment of the radially-oriented corona-emitting wire segments has to be performed, as thei tips must surpass by 1 to $3 \mathrm{~mm}$ the extremities of the tubular metallic supports. Anyhow, this operation is not as complicated and time-consuming than that of changing the needles of a conventional corona electrode.
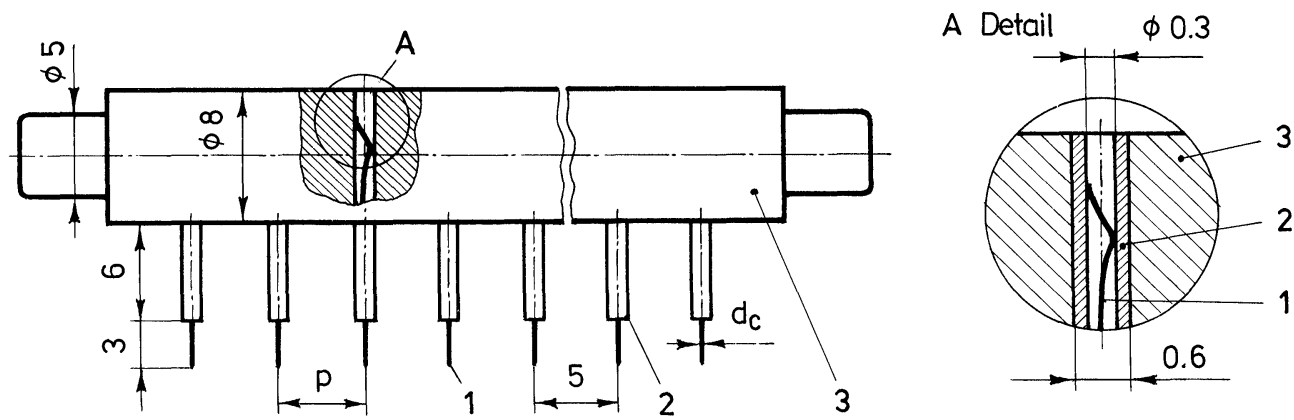

Fig. 4. A new type of charge neutralization electrode for hightension separators; 1 - radially-orientated corona-emitting wire segment; 2 - radially-orientated guiding metallic tube; 3 electrostatic support.

The intensity of the corona current is not affected by the wear of the wire segments, because the geometry of the emitting elements does not modify (cylinders of constant diameter). Thus, replacement of the corona-emitting elements might be necessary 
only after several thousands of working hours. This operation can be manually effectuated in a very short time (less than 10 minutes/ $1 \mathrm{~m}$ of electrode).

\section{DESIGN CONSIDERATIONS}

Laboratory testing of the new electrode design (Fig. 5) was considered necessary prior to manufacturing an industrial prototype. The experiments employed a reversibele d. c. highvoltage supply, fully-adjustable between 0 and $50 \mathrm{kV}, 6 \mathrm{~mA}$, an electrostatic voltmeter $0-10-20-30-50 \mathrm{kV}$, and a galvanometer $(0 \ldots 1000) \mu \mathrm{A}$.

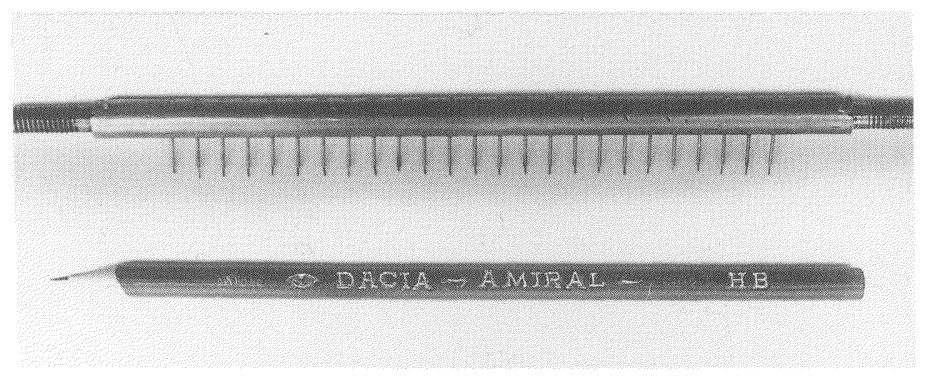

a

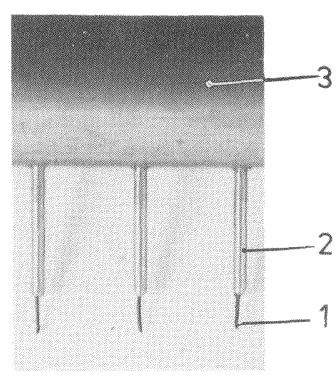

b

Fig. 5. Charge-neutralization electrode with radially-orientated corona-emitting wire segments (a); constructive detail (b); 1 radially-orientated corona-emitting wire segment; 2 - radiallyorientated guiding metallic tube; 3 - electrostatic support.

A first group of experiments has been performed in order to determine the optimum value of the distance $p$ between two adjacent corona-emitting elements. It has been found, examining the $\mathrm{I}(\mathrm{U})$ characteristics in Fig. 6 that there is no spectacular increase of corona current when reducing $\mathrm{p}$ below a certain limit (which might vary with $h$ ). This suggests that industrial wiper electrodes should not have more than 100 radially-orientated tubular metallic supports per meter.

The diameter of the metallic wire segments was thought to be an important design parameter of the electrode. Experiments shown that there is no significant difference between $\mathrm{I}(\mathrm{U})$ characteristics 
corresponding to $\varnothing 0.15 \mathrm{~mm}$ and to $\varnothing 0.25 \mathrm{~mm}$ wire segments, except for the somewhat lower corona on-set voltage observed in the former case. As the material of the wire might influence the long-term behaviour of the electrode, an experiment has been started on this topic at ELECTROMURES Co., Tirgu Mures, Romania, by the High-Intensity Electric Fields Research Laboratory of the Technical University of Cluj-Napoca, in order to compare wolfram-, molibden-, and steel-made corona-emitting elements.

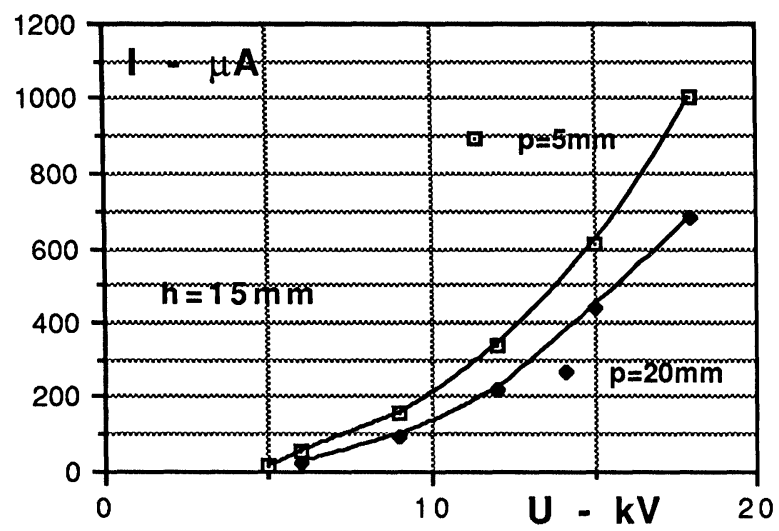

Fig. 6. Influence of $\mathrm{p}$ (distance between adjacent corona-emitting elements) on $\mathrm{I}(\mathrm{U})$ characteristics of the charge-neutralization electrode.

The rated voltage of the wiper electrode supply must be determined from an experiment which simulates the actual operating conditions in an industrial environement. The examination of $\mathrm{I}(\mathrm{U})$ characteristics in Fig. 7 reveals that the cleanliness of the surface of the opposed electrode influences to a great extent the density of the corona current I emitted by the wiper electrode at a given high-voltage $U$. The progressive corrosion of the electrodes subjected to corona discharges in room air [10] may modify the discharge parameters, but further researches are needed in order to produce conclusive evidence of this phenomenon. 


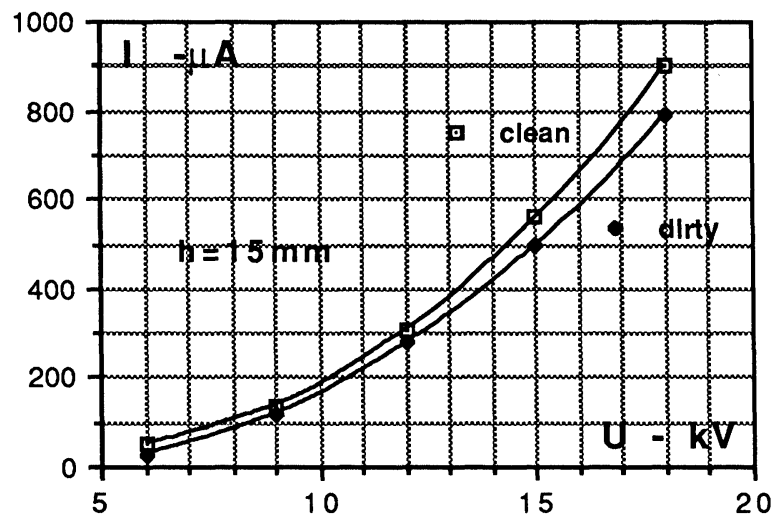

Fig. 7. Influence of opposing-electrode surface-state on $\mathrm{I}(\mathrm{U})$ characteristics of the charge-neutralization electrode.

The experiments have demonstrated that the corona discharge is also influenced by the presence of a mono-layer of granular electroinsulating material on the surface of the opposed electrode (Fig. 8).

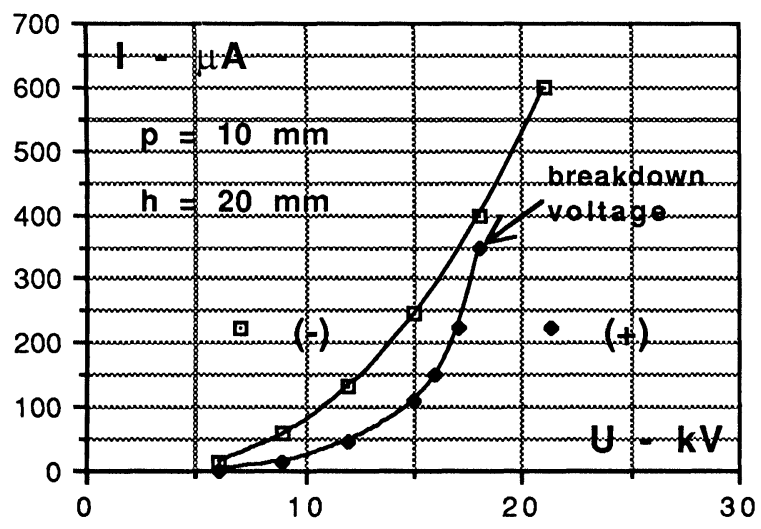

Fig. 8. I(U) characteristics of the charge-neutralization electrode in the absence (curve "-") and in the presence (curve "+") of a monolayer of electroinsulating granules on the surface of the opposing electrode. 
The corona on-set voltage is lower, because the dielectric layer determines the field intensification on the emitting tips of the electrode. At the same time, a considerable decrease of the breakdown voltage can be observed, as a consequence of higher field values in the air gap. Prior to breakdown, back corona appear from the earthed electrode, as local field intensification is caused by the layer of granular dielectric material (Fig. 9), a mechanism which is similar to that described in [11].

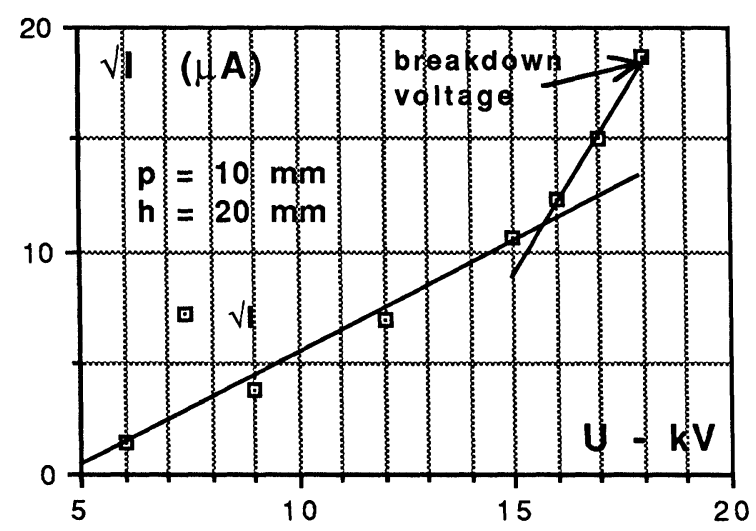

Fig. $9 \sqrt{ } \mathrm{I}(\mathrm{U})$ characteristic of the charge-neutralization electrode in the presence of a mono-layer of electroinsulating granules on the surface of the opposing electrode.(evidence of backcorona emission).

\section{ELECTRODE POSITION}

A larger distance $\mathrm{h}$ is more convenient than a smaller one, because: (i) back-corona and breakdown phenomena are less-likely to occur; (ii) material stream is less affected by the presence of the wiper electrode; (iii) corona-discharge zone is more extended; (iv) effective charge neutralization can be achieved even at high values of roll velocity. At the same time, there are two important drawbacks of wiper electrodes positioned too far away from the earthed roll-electrode: (i) this solution requires increased overall dimensions of the electroseparator; (ii) higher voltages are 
necessary, in order to ensure enough corona current for charge neutralization, as shown in Fig. 10.

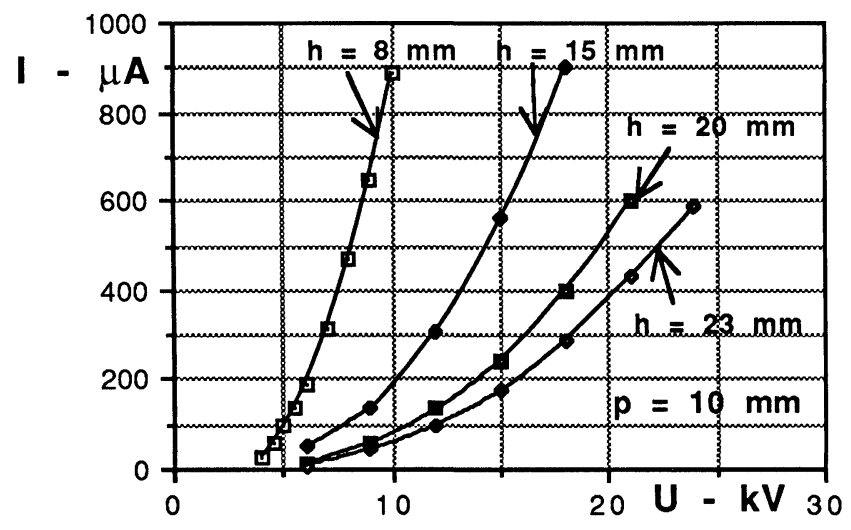

Fig. 10. Influence of charge-neutralization electrode position on its I(U) characteristics.

The optimum electrode position of the wiper electrode represents a compromise between these conflicting requirements. This is why a device that enables modification of electrode position is very useful with both laboratory and industrial electroseparators.

\section{CONCLUSIONS}

A topic usually neglected by the researchers of electroseparation processes, charge neutralization is nevertheless of utmost imprtance for the safe and efficient operation of the industrial installations. the residual charge of the particles may initiate a local breakdown when approaching a metallic wall and the electric image force determines the attachement of the granules on the metallic ducts, hindering the flow of the material. This is why the authors have tried to develop the theory of charge neutralization. Further researches are needed in order to optimize the frequence and the wave-form of the high-voltage energizing the charge-neutralization electrodes. 
The wiper electrodes employed to neutralize the residual charge of low-conductivity granules in a roll-type high-tension separator have to materialize various electrical, mechanical and technological requirements. The new corona design suggested in this paper seems to achieve a good performance/cost ratio. Its specific features: small cross-section (neglectable perturbation of the material stream), low corona on-set voltage, good stability of corona discharge during long-time operation, short maintenance time, have been tested on a laboratory model, prior to industrial application on ELSIM-2 insulation-metal separators, manufactured by ELECTROMURES co., Tirgu Mures, Romania.

Either d. c. or a. c. high-voltage generators may energize the charge-neutralization electrodes. Several criteria for electrode positioning have been discussed, but no general recommandation could be formulated.

The design considerations presented in this paper, based on systematic laboratiry testing, contributed to the optimisation of the new model of charge neutralization electrode, and could be extended to other types of electrodes or to other applications.

\section{ACKNOWLEDGEMENTS}

The authors express their thanks to eng. Lucian Pop and Mariana Olt, who have done the photographs, and to their former students, Crenguta Marza and Dorin Miere, who contributed to the manufacturing of the new electrode.

Many experiments have been done at Laboratoire d'Electrostatique et de Matériaux Diélectriques - C. N. R. S. Grenoble, where one of the authors (L. D.) spent 9 months with a Research Fellowship offered by the French Government.

\section{REFERENCES}

1. A.I. Mesenjasin, Elektriceskaja separacija $\mathrm{v}$ sil'nyh poljah (Nedra, Moskva, 1978). 
2. J.-F. Delon, Annales des mines, 3, 37 (1966).

3. F.S. Knoll, J.E. Lawver and J.B. Taylor, Ullmann's Encyclopedia of Industrial Chemistry (VCH, Weinheim, 1988) pp. 20-1 - 20-11, vol. B2.

4. B.R. Prabhakar, Geol. Surv. Ind. Spl. Pbl. No. 24, 211 (1989).

5. *** The Solution to Separation (Bulletin 904, Carpco Inc., U.S.A., 1990)

6. T. Meinander, Aufbereitungs Technik, nr.3, 127 (1977).

7 R. Morar, A. Iuga and L. Dascalescu, Modelling. Simulation \& Control - A, 14, 57 (1987).

8. A. Iuga, L. Dascalescu, R. Morar, I. Csorvasy and V. Neamtu, L Electrostatics, 23, 235 (1989).

9. L. Dascalescu, A. Iuga and R. Morar, Materials Science, 16, 273 (1990).

10. R. Le Ny, Int. Phys. Conf. Ser. No. 66: Session VI. Electrostatics 1983 Oxford, 173 (1983).

11. J..A. Cross and N.G. Barton, J. Electrostatics, 15, 15 (1984).

12. G. Pistre, Lournées d'études "Electrostatique", Poitiers, 41 (1992).

Lucian Dascalescu: See biography in previous paper.

Roman Morar: See biography in previous paper.

Alexandru Iuga: See biography in previous paper.

Vasile Neamtu obtained his M.Sc. degree in electromechanical engineering from the Technical University of Cluj-Napoca in 1975. His professional carrier began with Cluj Mining Company. since 1980 he has been at the Technical University of Cluj-Napoca where he lectures on electrotechnics and electrical machines. His main research interest is the development of high-voltage generators for electroseparators. 
Ilie Suarasan received his B.Sc. degree in electrical machines and his M.Sc. degree in electronics and telecommunications from the Technical University of Cluj-Napoca in 1978 and 1987, respectively. After 10 years with the R\&D department of Tehnofrig Co. he joined, in 1987, the Research Laboratory of High-Intensity Electrical Fields. He has been in charge of development of electronic control and of high-voltage generators for electroseparators.

Keywords: high-tension separator, corona electrode, image force, charge neutralization. 